\title{
BMJ Open Clinical values of FDG PET in polymyositis and dermatomyositis syndromes: imaging of skeletal muscle inflammation
}

\author{
Maki Tateyama, ${ }^{1,2}$ Kazuo Fujihara, ${ }^{1}$ Tatsuro Misu, ${ }^{1}$ Akira Arai, ${ }^{3}$ Tomohiro Kaneta, ${ }^{3}$ \\ Masashi Aoki ${ }^{1}$
}

To cite: Tateyama M, Fujihara K, Misu T, et al. Clinical values of FDG PET in polymyositis and dermatomyositis syndromes: imaging of skeletal muscle inflammation. BMJ Open 2015;5:e006763. doi:10.1136/bmjopen-2014006763

\section{- Prepublication history} material for this paper is available online. To view these files please visit the journal online (http://dx.doi. org/10.1136/bmjopen-2014006763).

Received 28 September 2014 Revised 28 October 2014 Accepted 21 November 2014

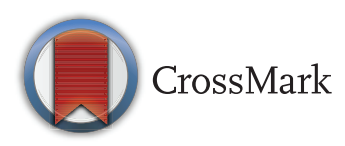

\footnotetext{
${ }^{1}$ Department of Neurology, Tohoku University School of Medicine, Sendai, Japan ${ }^{2}$ Department of Neurology, Iwate National Hospital, Iwate, Japan

${ }^{3}$ Department of Diagnostic Radiology, Tohoku University School of Medicine, Sendai, Japan
}

Correspondence to Dr Maki Tateyama; mtateyama@iwate-h.com

\begin{abstract}
Objectives: $\left[{ }^{18} \mathrm{~F}\right]$ Fluorodeoxyglucose positron emission tomography (FDG PET), a standard tool for evaluating malignancies, can also detect inflammatory lesions. However, its usefulness in evaluating muscle lesions in polymyositis and dermatomyositis syndromes (PM/DM) has not been established.

Methods: 33 patients with PM/DM who had undergone FDG PET were retrospectively analysed. FDG uptake was visually evaluated (visually identified FDG uptake, vFDG) in 16 regions of the body using mediastinum blood vessels as a positivity criterion. We also calculated the maximum standardised uptake value (SUVmax) in all four limbs of the patients with PM/DM as well as in 22 patients with amyotrophic lateral sclerosis (ALS) with similar disabilities. In 24 patients with PM/DM, MRI and FDG PET findings were compared.
\end{abstract}

Results: vFDG was observed in multiple muscle lesions with varying distributions in two-thirds of the patients with PM/DM, with most lesions being symmetrical. The number of vFDG-positive regions strongly correlated with the mean SUVmax in all four limbs $(p<0.0001)$. Histological grades of biopsied muscles correlated with both the mean SUVmax and number of vFDG-positive regions. Serum creatine kinase levels were higher in patients with more than two vFDG-positive regions than in those with two or less regions $(p<0.05)$. While the inflamed muscles showed diffused, patchy or marginal signal abnormalities on MRI, FDG uptake was most prominent inside the muscles. Compared with ALS, the mean SUVmax was significantly higher in the patients with PM/DM $(p<0.0001)$ and showed a striking correlation in the bilateral muscles, reflecting symmetrical muscle involvement in PM/DM. Conclusions: The visual assessment of FDG uptake as well as calculation of SUV enabled us to comprehensively evaluate skeletal muscle. This method can improve clinical practices and provide insights into pathomechanisms of PM/DM.

\section{INTRODUCTION}

Idiopathic inflammatory myopathies are a heterogeneous group of disorders clinically

\section{Strengths and limitations of this study}

- This is the first study that comprehensively investigated the usefulness of $\left[{ }^{18} \mathrm{~F}\right]$ fluorodeoxyglucose positron emission tomography (FDG PET) in evaluating muscle lesions in patients with polymyositis dermatomyositis syndromes using visual evaluation and standardised uptake value measurement. The study demonstrated the usefulness of these two methods. Visual evaluation of FDG uptake can be used in clinical practice.

- The limitation of the study is that it is retrospective. Patients underwent FDG PET for the detection of occult cancer; therefore, the imaging range was from the head to the middle of the thighs. Eight patients underwent FDG PET and 25 patients underwent FDG PET/CT. Data regarding manual muscle test were not obtained. Polymyositis dermatomyositis syndromes and non-inflammatory myopathies were not compared.

characterised by progressive proximal muscle weakness and pathologically by mononuclear cell infiltration and fiber necrosis in muscles. Polymyositis (PM), dermatomyositis (DM) and inclusion body myositis are representative phenotypes. ${ }^{1}$ Both PM and DM are thought to be immune-mediated disorders that can be successfully treated if properly managed. In contrast, in inclusion body myositis, degenerative processes also play an equal or greater role and effective treatments remain to be elucidated. $^{2}$ Patients with PM and DM syndromes may present with other organ involvement, such as interstitial lung disease. ${ }^{3} 4$ They may also evolve with other collagen diseases and malignancies. ${ }^{4}$ In addition, the extent and pattern of muscle involvement are variable. PM/DM can present with prominent truncal muscle weakness or preferential involvement of respiratory muscles. ${ }^{6} 7$ Thus, it is essential to systemically diagnose and evaluate patients with $\mathrm{PM} / \mathrm{DM}$. 
$\left[{ }^{18} \mathrm{~F}\right]$ Fluorodeoxyglucose positron emission tomography (FDG PET) is a standard tool for detecting malignancies. FDG also accumulates in inflammatory lesions where glucose-consuming inflammatory cells infiltrate. ${ }^{8}$ FDG PET is useful for diagnosing systemic inflammatory diseases, including collagen vascular disorders such as rheumatoid arthritis, vasculitis and polymyalgia rheumatica. $^{9-11}$ In PM/DM, only a limited number of studies have demonstrated FDG PET detection of inflammatory muscle lesions. Owada et al visually assessed FDG uptake (FDG uptake greater than or equal to that of the liver) in proximal muscles using FDG PET in 24 patients with PM/DM. They found that increased FDG uptake was more frequent in patients with $\mathrm{PM} / \mathrm{DM}$ than in controls (33\% vs $2 \%) .{ }^{12}$ Pipitone et al measured the maximum standardised uptake value (SUVmax) in the proximal muscles of all four limbs and calculated the muscle/liver SUVmax ratio in 12 patients with PM/DM using FDG PET/CT. They showed that the proximal muscle SUV ratio was higher in patients with $\mathrm{PM} / \mathrm{DM}$ than in controls. ${ }^{13}$ In these two studies, increased FDG uptake in proximal muscles did not correlate with clinical parameters or the MRI findings. In contrast, Tanaka et $a l^{14}$ measured the mean SUV using FDG PET/CT in 14 proximal muscle groups of 20 patients with $\mathrm{PM} / \mathrm{DM}$ and demonstrated that an increased SUV in proximal muscles of myositis patients as well as the mean proximal muscle SUV were correlated with serum creatine kinase (CK) and muscle strength. They also found that local SUV correlated with the degree of inflammation in muscle biopsies and weakness of the corresponding muscles. However, their method using global SUV calculations may not be feasible in daily clinical practice. For successful clinical application of FDG PET, a simple and reliable way to assess $\mathrm{PM} / \mathrm{DM}$ muscles is preferable.

In this study, we visually evaluated FDG PET findings in patients with $\mathrm{PM} / \mathrm{DM}$ in detail, and determined the extent and pattern of inflammation. We compared visually evaluated FDG PET findings with SUVmax in proximal muscles as well as with clinical and pathological findings. We also compared MRI findings with FDG uptake in the same muscle regions. Further, we compared SUVmax in proximal muscles between patients with $\mathrm{PM} / \mathrm{DM}$ and those with amyotrophic lateral sclerosis (ALS).

\section{MATERIALS AND METHODS \\ Patients}

33 patients with recent onset of PM/DM were enrolled in this study. Patients underwent FDG PET or FDG PET/CT to investigate malignancies before or shortly after receiving an initial corticosteroid treatment from January 2009 to July 2013. They were identified through a retrospective review of medical records in our department. Clinically, patients showed symmetrical proximal muscle weakness, elevated serum muscle enzymes and myositis-compatible electrophysiological findings proposed by Bohan and
Peter. ${ }^{15}$ Muscle biopsies were conducted in all patients and showed inflammatory infiltrates, muscle fibre necrosis and/or expression of HLA class 1 on muscle fibres. A few patients showed either inflammatory infiltrates or muscle fibre necrosis. In these patients, other muscle diseases were carefully excluded. Subsequent therapy with only corticosteroids or additional immunomodulatory therapies were effective in all but one patient who died before treatment initiation. Clinical records, laboratory data and muscle MRIs from each patient were collected. Patients with inclusion body myositis were not included. We identified 22 patients with ALS who were admitted to our hospital for diagnosis and had undergone FDG PET/CT for the detection of occult cancer. These patients were included in this study as disease controls. Eighteen patients fulfilled the diagnostic criteria for clinically definite, clinically probable or clinically probable laboratory supported ALS (according to the revised El Escorial criteria of the World Federation of Neurology). ${ }^{16}$ Four patients showed only lower motor neuron symptoms with exclusion of other diseases. Degrees of disability in patients with $\mathrm{PM} / \mathrm{DM}$ and those with ALS were similar; all these patients were able to walk and performed activities of daily life independently, but with some difficulty.

\section{FDG PET imaging}

Patients fasted for a minimum of $4 \mathrm{~h}$ before the ${ }^{18} \mathrm{~F}$-FDG injection. Blood glucose levels were measured and patients with blood levels $>150 \mathrm{mg} / \mathrm{dL}$ were not included. After injection of approximately $185 \mathrm{MBq}$ $(3.1 \mathrm{MBq} / \mathrm{kg})$ of ${ }^{18} \mathrm{~F}-\mathrm{FDG}$, the patients rested on the bed for $1 \mathrm{~h}$. PET scan was then performed from the head to mid-thigh using a PET scanner (ECAT EXACT HR+, Siemens, Erlangen, Germany) or PET/CT scanner (Biograph Duo or 40, Siemens, Erlangen, Germany). PET/CT scanners have been used since April 2009 and have replaced a much-used PET scanner in our institute. FDG uptake was visually evaluated (visually identified FDG uptake, vFDG) in skeletal muscles by two radiologists (AA and TK). FDG uptake was independently assessed in a blinded manner. vFDG was evaluated in 16 regions - including the upper arms; shoulders; sternocleidomastoid muscles; paraspinal muscles of cervical, thoracic and lumbar levels; buttocks and upper part of the thighs, on both sides (figure 1). Regions with an FDG uptake greater than or equal to that of the mediastinum blood vessels were considered vFDG-positive. ${ }^{17}$ Regions judged to be positive by both radiologists were defined as vFDG-positive and the number of vFDG-positive regions were counted (minimum $=0$ and maximum $=16$ in each patient). In addition, SUV was calculated in patients with PM/DM and in 22 patients with ALS in the biceps brachii, quadriceps femoris, and medial and posterior compartments of the thigh, on both sides. In 1 patient with PM, we were not able to calculate SUV in the biceps brachii, because the sectional area of the muscle was of insufficient size for 


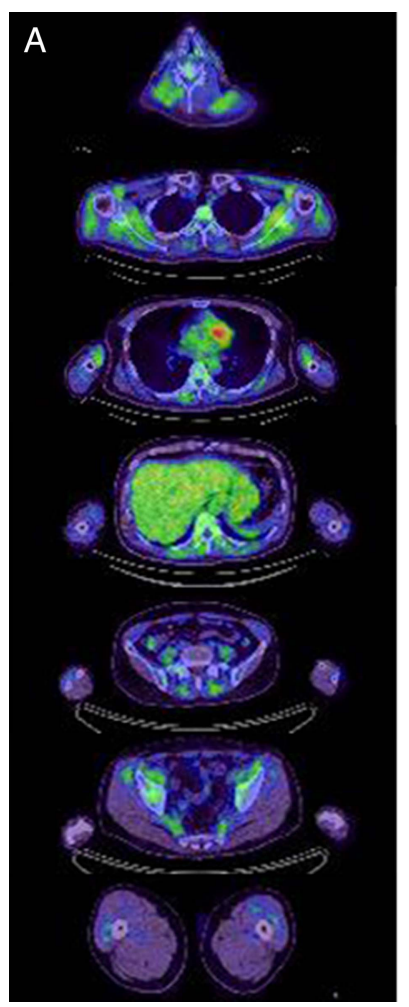

B

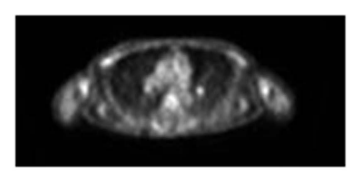

C

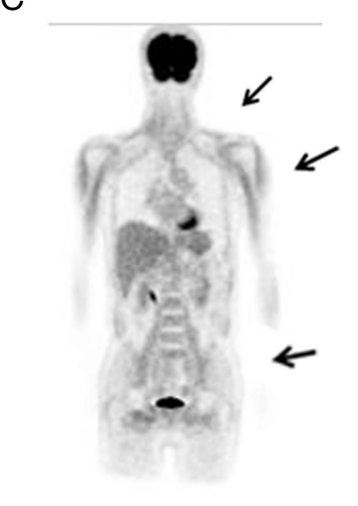

D

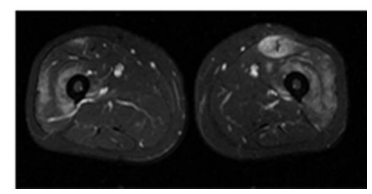

Figure $1\left[{ }^{18} \mathrm{~F}\right]$ fluorodeoxyglucose positron emission tomography (FDG PET) and MRI findings in a patient with dermatomyositis (patient 11). Serial sections of FDG PET/CT (A). Using FDG uptake in mediastinum blood vessels as a positivity criterion, positive regions were found in the paraspinal muscles, shoulders, upper arms and lumbar girdles in a predominantly symmetrical distribution. An FDG PET image of the upper arm level (B). Frontal view of an FDG PET image indicating FDG uptake in the shoulders, upper arms and iliopsoas (C). MRI of the thighs (D) showing high signal areas in the bilateral quadriceps femoris (T2-weighted images with fat suppression).

placement in the region of interest (ROI). The ROI $(20 \mathrm{~mm})$ was placed in the highest FDG uptake area in each muscle region. SUV was calculated as both the maximum value (SUVmax) and mean value (SUVmean) in ROIs. The mean proximal muscles SUVmax (mean SUVmax) and SUVmean (mean SUVmean) were calculated by averaging the values obtained for the six muscle regions.

\section{Muscle MRI}

Muscle MRI was performed during a routine examination using the $1.5 \mathrm{~T}$ Intera scanner (Philips, Best, the Netherlands) before treatment. Results were assessed by experienced radiologists. Abnormal signals in skeletal muscles were identified on T2-weighted images with fat suppression or short $\tau$ inversion recovery sequence with long TE. In the patients who also underwent gadolinium contrast enhancement, abnormal enhancement in the muscle and/or muscle fascia were evaluated.

\section{Muscle biopsy}

Muscle biopsies were obtained before treatment in all patients with PM/DM. The muscle was taken from the biceps brachii (26 patients), deltoid (3 patients), quadriceps femoris (3 patients) and gastrocnemius (1 patient). Biopsied muscles were snap frozen and a routine histochemical study was conducted. The most affected lesions in each specimen were photographed with objective lens of 10-fold magnifications. Histological findings were assessed in a blinded manner by an experienced neurologist well versed in neuropathology (TM). The extent of mononuclear cell infiltration (mononuclear cell infiltration score) was graded as follows- 0 : none or slight; 1 : one focus of mononuclear cell infiltration; 2: more than one focus of mononuclear cell infiltration; and 3: diffuse mononuclear cell infiltration. Muscle fibre necrosis and regeneration (necrosis/regeneration score) were graded as follows: 0 : none; $1: 1 \%$ or less of muscle fibres showing necrosis or regeneration; 2: more than $1 \%$ and no more than $10 \%$ of muscle fibres showing necrosis or regeneration; and 3: more than $10 \%$ of muscle fibres showing necrosis or regeneration. The total histological scores were calculated for each patient by adding the mononuclear cell infiltration and the necrosis/regeneration scores.

\section{Statistical analysis}

Statistical analysis was performed using JNP8 software (SAS Institute Inc., Cary, North Carolina, USA). Statistical significance was analysed by the Wilcoxon rank-sum test (non-parametric), simple regression analysis and Spearman rank correlation. Comparisons were considered to be statistically significant if $\mathrm{p}<0.05$.

\section{RESULTS}

\section{Patient characteristics}

The characteristics of the 33 patients with PM/DM (10 males, 23 females; mean age $56 \pm 17.9$ years) who underwent FDG PET (8 patients) or FDG PET/CT (25 patients) are summarised in table 1. FDG PET was performed before any treatment in 25 patients. In 8 patients, FDG PET was performed shortly after beginning corticosteroid treatment (2-9 days; mean 6.1 days). We provisionally divided patients with $\mathrm{PM} / \mathrm{DM}$ according to the presence or absence of other collagen diseases. The number of patients in each clinical group and those with relevant clinicopathological classifications (as proposed in the 119th European Neuromuscular Centre international workshop) ${ }^{18}$ in each clinical group (parenthesis) were as follows: 11 patients with DM without other collagen diseases (4 patients with definite $\mathrm{DM}$ and 7 with probable DM), 11 patients with PM without other collagen diseases (9 patients with nonspecific myositis, 1 with definite PM and 1 with probable $\mathrm{PM})$, and 11 patients with PM/DM with other collagen diseases (8 patients with non-specific myositis, 2 with probable PM and 1 with definite PM). 2 patients with DM and 1 with PM without other collagen diseases were 


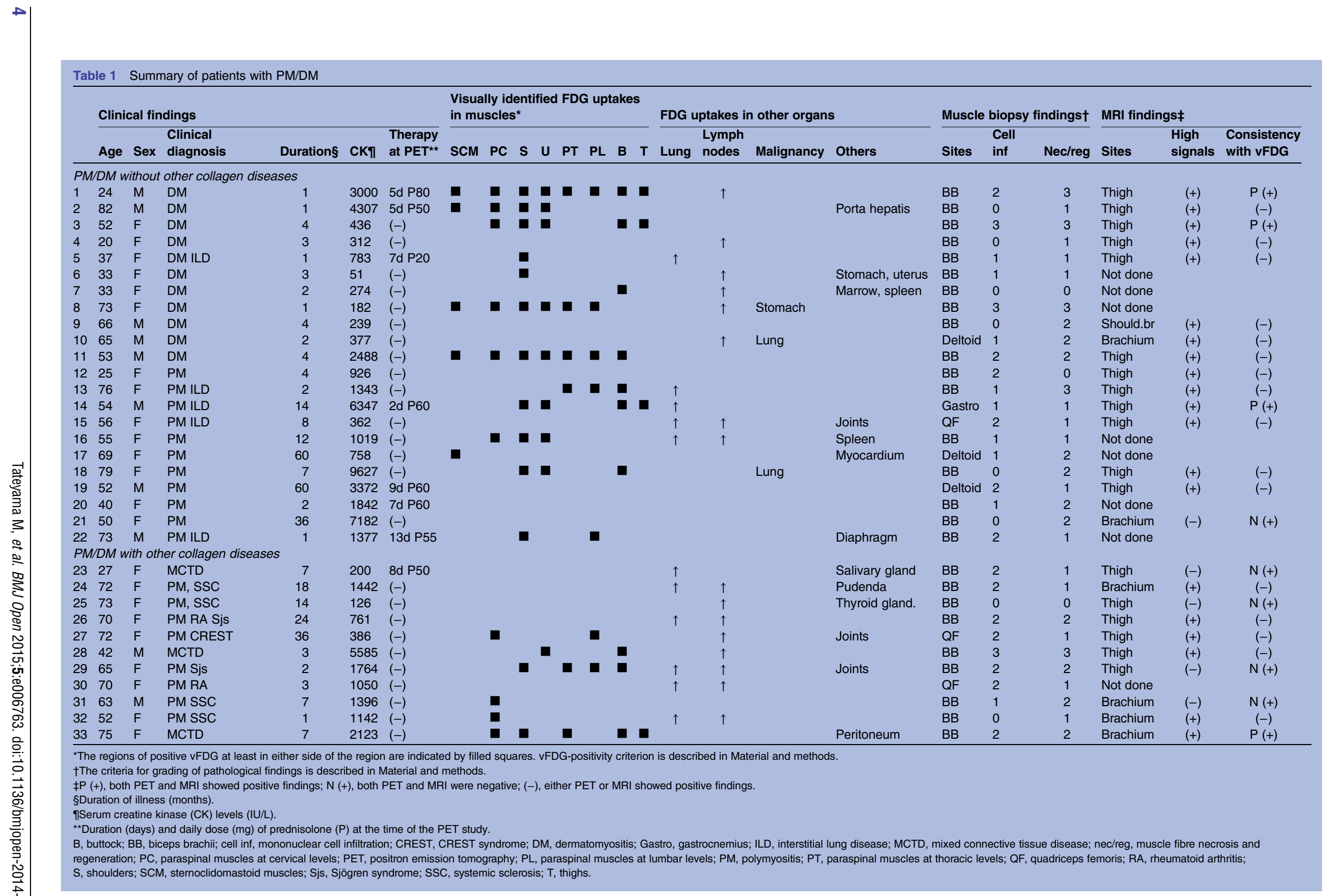


shown to have malignancies. Abnormal FDG uptake was noted in the lungs in several patients, and most were diagnosed as having interstitial lung diseases. FDG uptake in the lymph nodes was observed in $50 \%$ of patients with DM, $18.2 \%$ of patients with $\mathrm{PM}$ without other collagen diseases and $75 \%$ of patients with PM/ DM with other collagen diseases.

\section{Visual assessment of FDG uptake in skeletal muscles of patients with PM/DM}

vFDG in skeletal muscles was observed in 20/33 patients with $\mathrm{PM} / \mathrm{DM}(60.6 \%)$. vFDG was detected in multiple regions in 14 patients $(42.4 \%)$ with various patterns (table 1) and almost symmetrical distribution. The shoulders and buttocks were the most frequent vFDG-positive regions. A fraction of patients with DM showed vFDG-positivity in most of the regions: a representative case is shown in figure 1 . The number of vFDG-positive regions correlated with the mean SUVmax of all four extremities $(\mathrm{r}=0.87, \mathrm{p}<0.0001$; figure $2 \mathrm{~A})$, implying that SUVmax in extremities could be inferred by the extent of vFDG-positive regions. There was no correlation between serum CK levels and the number of vFDG-positive regions $(p=0.20)$. However, serum CK levels were higher in patients with more than two vFDG-positive regions than in those with two or less vFDG-positive regions $(\mathrm{p}=0.0179$; figure 2B). The number of vFDG-positive regions also correlated with the total histological score $(\mathrm{r}=0.49, \mathrm{p}=0.0038$; figure 2C). The number of vFDG-positive regions correlated with necrosis/regeneration scores $(\mathrm{r}=0.49, \mathrm{p}=0.0036)$, but not with the mononuclear cell infiltration scores $(\mathrm{p}=0.06)$.

\section{Relationship between SUV and clinicopathological findings in patients with PM/DM}

The mean SUVmax did not correlate with the duration of the illness. The mean SUVmax was higher in men than in women, although not significant $(\mathrm{p}=0.075)$. There were no significant differences among patients with DM without other collagen diseases, PM without other collagen diseases and PM/DM with other collagen diseases. The total histological score correlated with the mean SUVmax $(\mathrm{r}=0.60, \mathrm{p}=0.0002$; figure 2D). The mean SUVmax correlated more strongly with necrosis/regeneration scores $(\mathrm{r}=0.61, \mathrm{p}=0.0002)$ than with mononuclear cell infiltration scores $(\mathrm{r}=0.40, \mathrm{p}=0.0198)$. The total pathological score also correlated with SUVmax in the biopsied muscles in the patients with $\mathrm{PM} / \mathrm{DM}$, in whom biopsies were performed in biceps brachii or quadriceps femoris $(\mathrm{r}=0.58, \mathrm{p}=0.0017)$. SUVmax in the biopsied muscles correlated more strongly with necrosis/regeneration scores $(\mathrm{r}=0.59, \mathrm{p}=0.0013)$ than mononuclear cell infiltration scores $(\mathrm{r}=0.40, \mathrm{p}=0.0361)$. On the other hand, the mean SUVmax did not correlate with serum CK levels. Serum CK levels did not correlate with total histological scores $(\mathrm{p}=0.08)$, necrosis/regeneration scores $(\mathrm{p}=0.22)$ and mononuclear cell infiltration scores $(\mathrm{p}=0.32)$.

\section{Comparison of FDG PET and MRI findings in PM/DM}

25 patients with PM/DM underwent both FDG PET and muscle MRI. Muscle MRI was performed for the thighs (18 patients), upper arms (6 patients) and shoulders (1 patient). 20 patients were judged to be MRI positive. In the MRI-positive regions of each patient, $\mathrm{vFDG}$ was positive in 4 patients. There were no patients who were MRI-negative and vFDG-positive. There was no difference in SUVmax of the muscles between MRI-positive and MRI-negative muscles ( $p=0.1537$; figure 2E). In contrast, SUVmax of relevant muscles was significantly higher in vFDG-positive patients than in vFDG-negative patients $(p=0.0076$; figure $2 F)$. These findings suggest that MRI is a sensitive tool for detecting inflammatory oedema. However, high signals on MRI may not simply reflect the degree of inflammation.

Interestingly, the pattern of abnormal signals was different between FDG PET and MRI (figures 1 and 3). On MRI, affected muscles showed several patterns including diffused or patchy high signals and high signals surrounding the muscles, which may reflect inflammatory oedema (figures 1 and 3). In contrast, FDG uptake was more localised in each muscle for most patients. The highest uptake was predominantly within muscles, a few centimetres from the muscle surface.

\section{SUVmax in the skeletal muscles of patients with PM/DM and ALS}

The mean SUVmax was higher in patients with PM/DM than in those with ALS $(1.463 \pm 0.483$ vs $1.004 \pm 0.136$, $\mathrm{p}<0.0001$; figure $4 \mathrm{~A})$. The mean SUVmean was also higher in patients with $\mathrm{PM} / \mathrm{DM} \quad(1.106 \pm 0.370$ vs $0.733 \pm 0.139, \mathrm{p}<0.0001)$.

The SUVmax in the biceps brachii, quadriceps femoris, and medial and posterior compartments of the thigh (both sides) was compared. In patients with PM/ DM, SUVmax of one muscle region and that of another muscle region was correlated (biceps brachii vs medial and posterior compartments of the thigh; quadriceps vs biceps brachii; quadriceps vs medial and posterior compartments of the thigh). Moreover, there was a striking correlation between the same muscles on both sides (biceps brachii, quadriceps femoris, medial and posterior compartments of the thigh). The scatter plots suggested symmetrical muscle involvement in $\mathrm{PM} / \mathrm{DM}$ (figure 4B). Only moderate symmetry in SUVmax was found in patients with ALS (figure 4C).

\section{DISCUSSION}

In the present study, we assessed the skeletal muscles of patients with PM/DM using FDG PET in two ways: visual assessment and SUV measurement. We found that the mean SUVmax in four extremities of patients with PM/ DM was approximately 1.5 and the mean SUVmean was approximately 1.1 . These results are consistent with those of previous studies. ${ }^{13} 14$ While a major mechanism underlying FDG accumulation in the inflamed tissue 
A

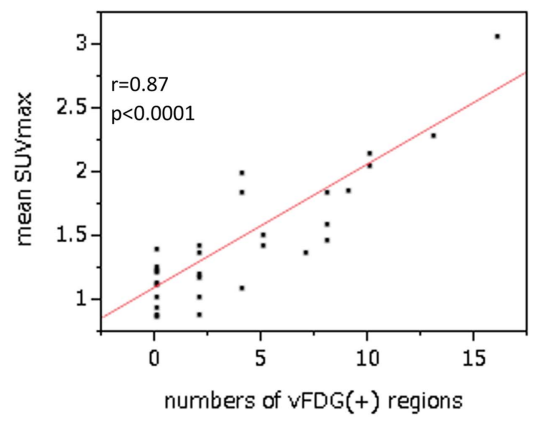

C
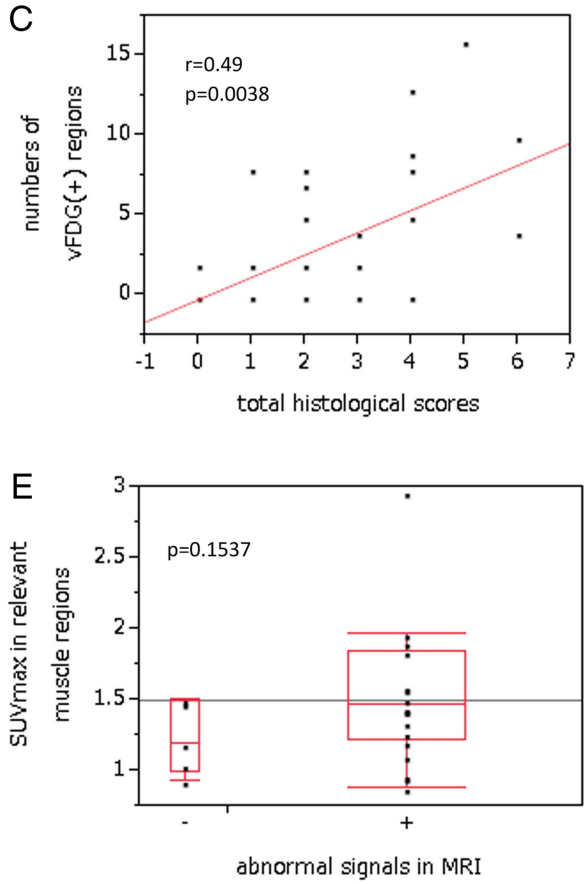

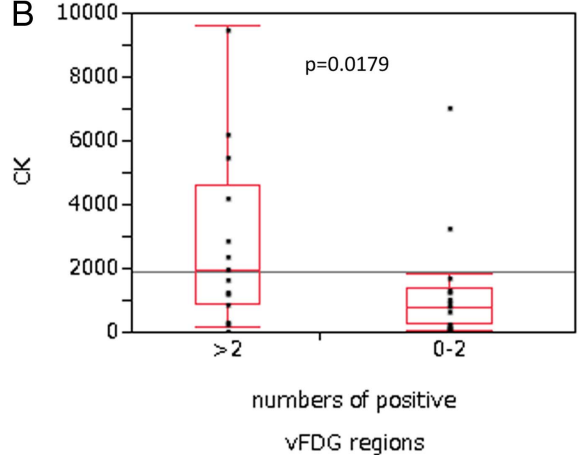

D
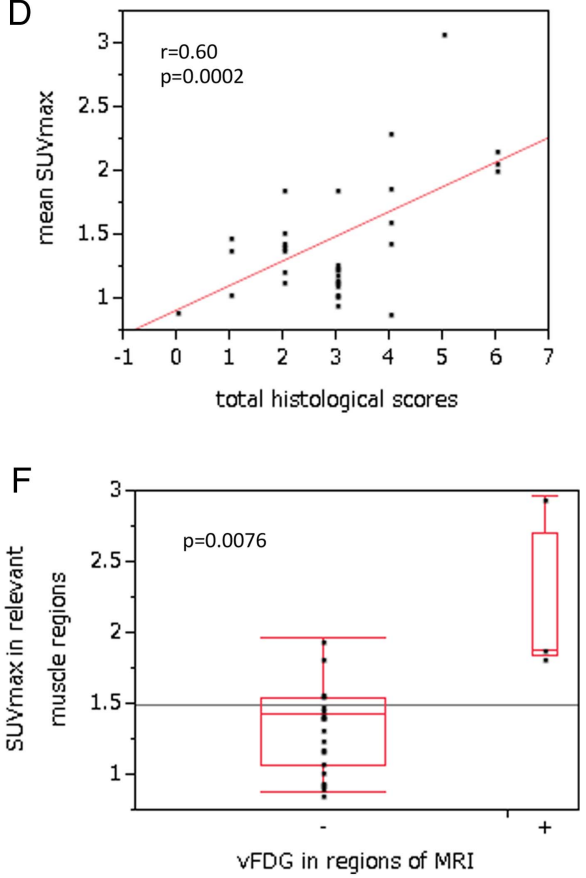

Figure 2 The numbers of visually identified fluorodeoxyglucose (vFDG)-positive muscle regions are correlated with the mean maximum standardised uptake value (SUVmax) in four extremities (A). Serum values of creatine kinase (CK) were higher in patients with more than two vFDG-positive muscle regions than in those with two or less regions (B). The number of vFDG-positive muscle regions correlated with the total histological scores (C). The mean SUVmax in four extremities correlated with the total histological scores (D). There were no differences in SUVmax in corresponding muscles between muscles with abnormal MRI signals and those without (E). In muscle regions examined by MRI, SUVmax in the corresponding muscles was higher in vFDG-positive muscles than in vFDG-negative ones (F).

caused by rheumatoid arthritis is uptake by metabolically active cells such as macrophages, young granulation tissue and fibroblasts, ${ }^{8} 9$ there has been no report on PM/DM. In the present study, we found that SUVmax of biopsied muscles correlated more strongly with necrosis/regeneration scores than with mononuclear cell infiltration scores. These findings imply that macrophages assembled in necrotic muscle fibre lesions and muscle fibres in regeneration stage may be a major background of FDG uptake in PM/DM.

Because FDG uptake in inflamed muscles is fairly moderate compared with that in the neoplasm, setting an appropriate criterion for visual assessment is challenging. In a previous study using liver in which an SUV of approximately 3 was the positivity criterion, $33 \%$ patients with PM/DM had FDG-positive muscle regions. ${ }^{12}$ In the present study, we chose the mediastinum blood vessels, where SUV is approximately 2, as a positivity criterion for $\mathrm{vFDG} .{ }^{17}$ We found that $60.6 \%$ patients with $\mathrm{PM} / \mathrm{DM}$ had vFDG-positive muscle regions. In addition, we divided the proximal body muscle into 16 regions, which enabled us to evaluate the extent and patterns of vFDG-positive regions. Most notably, the extent of vFDGpositive regions correlated with the mean SUVmax and histological findings. There was only a mild correlation between the extent of vFDG-positive regions and serum CK levels. Serum CK levels are a major clinical parameter in PM/DM; however, it did not correlate with pathological severity in our patients. These findings suggest that vFDG, using mediastinum blood vessels as the positivity criterion, is useful for the assessment of systemic muscle lesions and that the extent of vFDG-positive 


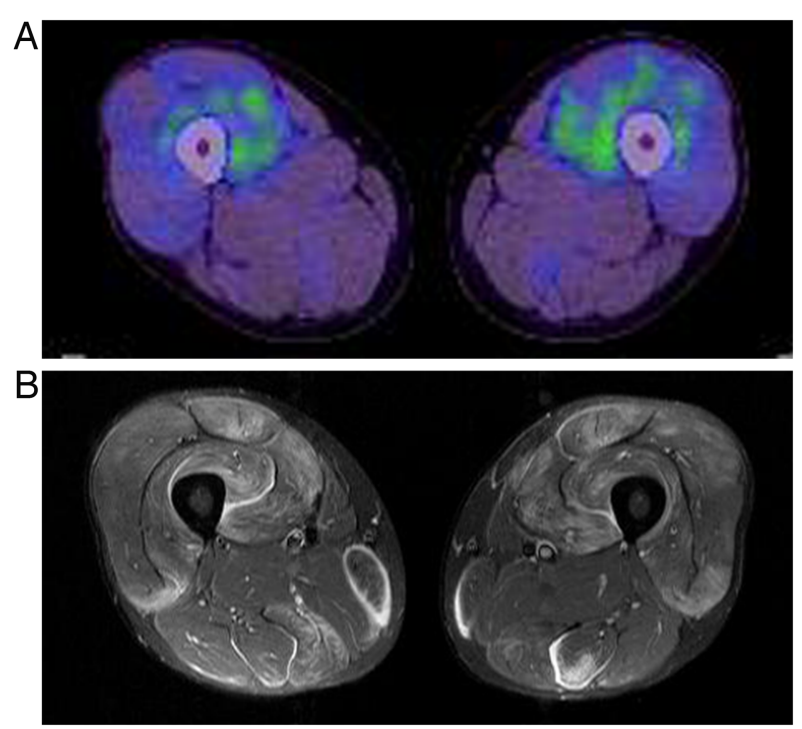

Figure $3 \quad\left[{ }^{18} \mathrm{~F}\right]$ fluorodeoxyglucose positron emission tomography (FDG PET) (A) and MRI findings (B) for both thighs in a patient with polymyositis (patient 14). Distribution patterns of high signal on MRI and FDG PET are different. The FDG uptake is localised and predominantly within the muscles.

regions can be an indicator of the disease activity in PM/DM.

We also evaluated vFDG using another scoring system, in which one point was assigned when the FDG uptake was greater than or equal to that of mediastinum blood vessels and two points assigned when FDG uptake was greater than or equal to that of the liver. The points given by two examiners were added in all the muscle regions in each patient (scores ranged from 0 to 64). These scores also strongly correlated with the mean SUVmax $(\mathrm{r}=0.90, \mathrm{p}<0.0001)$ and total histological scores $(\mathrm{p}=0.0014)$.

MRI is now widely used to diagnose and determine biopsy sites in PM/DM. ${ }^{19}{ }^{20}$ It can sensitively detect muscle oedema in diseased muscle, including inflammatory myopathies. ${ }^{21}$ Because MRI detects inflammatory oedema and FDG PET detects FDG uptake by metabolically active cells, the images produced by the two modalities are different. In fact, MRI-positivity did not correlate with SUVmax in the same muscles in the present study. On MRIs, abnormal signals were seen as diffused or patchy patterns in the muscles, and sometimes found surrounding the muscles. On the other hand, FDG uptake tended to localise and was frequently found deep within each muscle. Because FDG uptake theoretically reflects metabolically active sites, the pathology of the biopsied muscles may not represent the most affected lesions. This may be the reason why we only found a moderate correlation between the grade of pathological findings and SUVmax of corresponding muscles. In the present study, abnormal MRI signals were positive in 20 of the 25 patients, while vFDG was positive in 4; therefore, the sensitivity of MRI seems to be superior. However, MRI was not evaluated in a blinded manner in this study and comparison was only performed for MRI examination sites; other sites such as the paraspinal muscles and buttocks were not compared. Further investigation is required to clarify the precise nature of abnormal signals using these two measures.

Previous reports have shown that SUV in skeletal muscles is higher in patients with $\mathrm{PM} / \mathrm{DM}$ than in control patients without disability. ${ }^{13}{ }^{14}$ Comparisons of SUV between patients with $\mathrm{PM} / \mathrm{DM}$ and disabled control patients have never been reported. ALS is characterised by progressive muscle weakness caused by degeneration of the upper and lower motor neurons. We found that the mean SUVmax was significantly higher in patients with PM/DM than in those with ALS. These findings suggest that FDG PET can distinguish between muscle weakness resulting from muscle fibre destruction with inflammation and that resulting from neurogenic atrophy.

$\mathrm{PM} / \mathrm{DM}$ is clinically characterised by symmetrical proximal muscle weakness. ${ }^{4}$ In the present study, we found a strong correlation between SUVmax of the same muscles on both sides - including bilateral quadriceps femoris, bilateral medial and posterior compartments of the thighs, and bilateral biceps brachii. The correlation coefficient was higher for quadriceps femoris (both sides) and medial and posterior compartments of the thigh (both sides) than in quadriceps femoris and medial and posterior compartments on the same side of the legs. These findings statistically verified that the inflammatory muscle damage progresses symmetrically in PM/DM, although muscle lesions are often multifocal in each muscle. The current pathological mechanism of $\mathrm{PM} / \mathrm{DM}$ based on immunopathology cannot explain the symmetrical muscle damage in PM/ DM. ${ }^{42}$ Several possible mechanisms may explain the symmetry, such as an involvement of anatomical factors, including vasculatures and peripheral nerves, or some immune or physiological factors of individual muscles that can influence the extent of inflammation.

The present study has several limitations. The study was retrospective and FDG PET was performed to detect malignancies. The extent of the imaging range was, therefore, restricted from the head to the middle of the thighs, limiting evaluation of the thighs; however, a negligible number of false negatives in $\mathrm{vFDG}$ were most likely produced. Nevertheless, we propose that when FDG PET is conducted in patients with PM/DM, scans should include, at least, the entire length of the thighs to produce more informative data. In this study, we could not study whether FDG uptake in skeletal muscles changed after immunomodulatory treatment, because only a few patients underwent FDG PET two times or more. A part of patients underwent FDG PET shortly after beginning corticosteroid. Manual muscle test (MMT) data were not obtained from all patients; therefore, we could not compare MMT and FDG PET findings. We were unable to compare FDG PET findings 


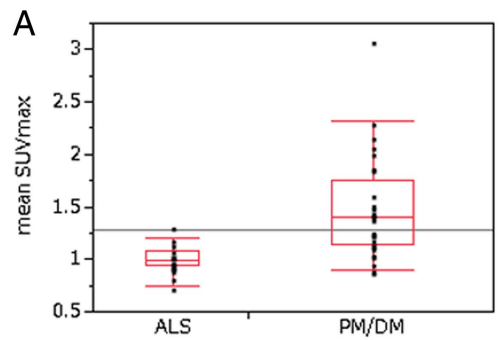

B

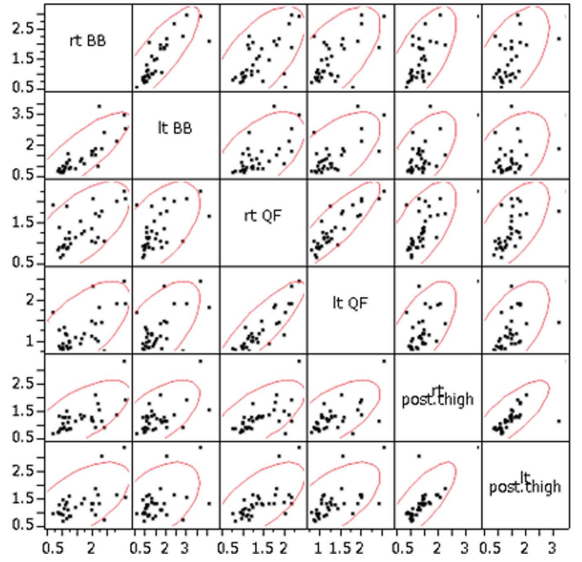

C

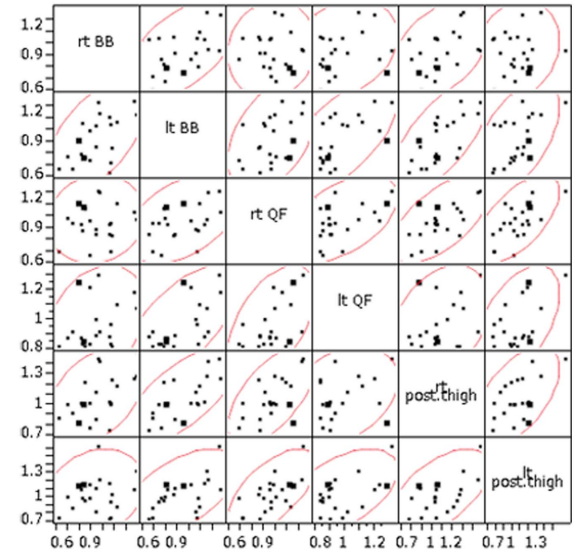

Figure 4 The mean maximum standardised uptake value (SUVmax) in four extremities was higher in patients with polymyositis and dermatomyositis $(P M / D M)$ than in those with amyotrophic lateral sclerosis $(A L S)(p<0.0001)(A)$. In patients with PM/DM, SUVmax of bilateral muscles was highly correlated, suggesting symmetrical muscle lesions (B). Correlation coefficients: bilateral quadriceps femoris, $r=0.91, p<0.0001$; bilateral medial and posterior compartments of the thighs, $r=0.88, p<0.0001$; right quadriceps femoris and right medial and posterior compartments of the thighs, $r=0.58, p=0.0004$; left quadriceps and left medial and posterior compartments of the thighs, $r=0.69, p<0.0001$. In patients with ALS, only a moderate correlation was found between bilateral muscles (Spearman rank correlation) (C).

between patients with $\mathrm{PM} / \mathrm{DM}$ and non-inflammatory myopathies, such as muscular dystrophy.

In conclusion, our findings indicated the utility and convenience of FDG PET in the clinical characterisation of $\mathrm{PM} / \mathrm{DM}$. The greatest advantage of FDG PET is that it can screen the whole body in a single scan. Apart from malignancies ${ }^{23}$ these methods can also evaluate lung inflammation $^{24}$ and swelling of lymph nodes. We can visually evaluate the extent and pattern of muscle lesions systemically and include structures that are not routinely screened by MRI. In addition, the degree of pathology in muscle can be inferred from the extent of vFDG-positivity. Semiquantitiative evaluation using SUV may be useful for the statistical analysis of muscle inflammation. On the other hand, the disadvantages of FDG PET include the considerable costs incurred and exposure to ionising radiation. It is important to keep in mind that FDG uptake in muscles is influenced by hyperglycaemia, uptake by other organs, and voluntary or involuntary muscle movement during the uptake phase; therefore, careful examination is a prerequisite. ${ }^{25}$ Although further prospective investigations in a larger sample size are necessary, addition of FDG PET to conventional clinical examinations may be useful for comprehensive diagnosis and management as well as investigation of the pathological mechanisms underlying $\mathrm{PM} / \mathrm{DM}$.
Acknowledgements The authors would like to thank Risa Ando for her excellent technical assistance. They would like to thank Climson (Enago) Interactive for their English language review.

Contributors MT, KF and TK were involved in the study setup and draft writing. TM was involved in the analysis of the muscle biopsies. TK and AA contributed to the visual evaluation of FDG PET. All of the authors contributed to discussion of the data, and review and revision of the manuscript.

Funding This study was supported by JSPS KAKENHI Grant Number 25461265 (Japan).

Competing interests None.

Ethics approval Tohoku University School of Medicine ethics committee.

Provenance and peer review Not commissioned; externally peer reviewed.

Data sharing statement No additional data are available.

Open Access This is an Open Access article distributed in accordance with the Creative Commons Attribution Non Commercial (CC BY-NC 4.0) license, which permits others to distribute, remix, adapt, build upon this work noncommercially, and license their derivative works on different terms, provided the original work is properly cited and the use is non-commercial. See: http:// creativecommons.org/licenses/by-nc/4.0/

\section{REFERENCES}

1. Lazarou IN, Guerne PA. Classification, diagnosis, and management of idiopathic inflammatory myopathies. J Rheumatol 2013;40:550-64.

2. Machado P, Brady S, Hanna MG. Update in inclusion body myositis. Curr Opin Rheumatol 2013;25:763-71.

3. Vij R, Strek ME. Diagnosis and treatment of connective tissue disease-associated interstitial lung disease. Chest 2013;143:814-24. 
4. Engel AG, Hohlfeld R. The polymyositis and dermatomyositis syndromes. In: Engel AG, Franzini-Armstrong C, eds. Myology. 3rd edn. McGraw-Hill, 2004:1321-66.

5. Tansley $\mathrm{S}$, Gunawardena $\mathrm{H}$. The evolving spectrum of polymyositis and dermatomyositis-moving towards clinicoserological syndromes: a critical review. Clin Rev Allerg Immunol 2014;47:264-73.

6. Maeda MH, Tsuji S, Shimizu J. Inflammatory myopathies associated with anti-mitochondrial antibodies. Brain 2012; 135(Pt 6):1767-77.

7. Tanaka K, Sato A, Kasuga K, et al. Chronic myositis with cardiomyopathy and respiratory failure associated with mild organspecific autoimmune diseases. Clin Rheumatol 2007;26:1917-19.

8. Gotthardt M, Bleeker-Rovers CP, Boerman OC, et al. Imaging of inflammation by $\mathrm{PET}$, conventional scintigraphy, and other imaging techniques. J Nucl Med 2010;51:1937-49.

9. Kubota K, Ito K, Morooka M, et al. FDG PET for rheumatoid arthritis: basic considerations and whole-body PET/CT. Ann N Y Acad Sci 2011;1228:29-38.

10. Fuchs M, Briel M, Daikeler T, et al. The impact of ${ }^{18} \mathrm{~F}-\mathrm{FDG}$ PET on the management of patients with suspected large vessel vasculitis. Eur J Nucl Med Mol Imaging 2012;39:344-53.

11. Yamashita $\mathrm{H}$, Kubota $\mathrm{K}$, Takahashi $\mathrm{Y}$, et al. Whole-body fluorodeoxyglucose positron emission tomography/computed tomography in patients with active polymyalgia rheumatica: evidence for distinctive bursitis and large-vessel vasculitis. Mod Rheumatol 2012;22:705-11.

12. Owada T, Maezawa R, Kurasawa K, et al. Detection of inflammatory lesions by $\mathrm{F}-18$ fluorodeoxyglucose positron emission tomography in patients with polymyositis and dermatomyositis. J Rheumatol 2012;39:1659-65.

13. Pipitone N, Versari A, Zuccoli G, et al. ${ }^{18} \mathrm{~F}$-fluorodeoxyglucose positron emission tomography for the assessment of myositis: a case series. Clin Exp Rheumatol 2012;30:570-3.

14. Tanaka S, Ikeda K, Uchiyama $\mathrm{K}$, et al. $\left[{ }^{18} \mathrm{~F}\right] \mathrm{FDG}$ uptake in proximal muscles assessed by PET/CT reflects both global and local muscular inflammation and provides useful information in the management of patients with polymyositis/dermatomyositis. Rheumatology 2013;52:1271-8.

15. Bohan A, Peter JB. Polymyositis and dermatomyositis. $N$ Engl J Med 1975;292:344-7.

16. Brooks BR, Miller RG, Swash M, et al. El Escorial revisited: revised criteria for the diagnosis of amyotrophic lateral sclerosis. ALS Motor Neuron Disord 2000;1:293-9.

17. Sobic-Saranovic DP, Grozdic IT, Videnovic-Ivanov J, et al. Responsiveness of FDG PET/CT to treatment of patients with active chronic sarcoidosis. Clin Nucl Med 2013;38:516-21.

18. Hoogendijk JE, Amato AA, Lecky BR, et al. 119th ENMC international workshop: trial design in adult idiopathic inflammatory myopathies, with the exception of inclusion body myositis, 10-12 Octber 2003, Naarden, The Netherland. Neuromusc Disord 2004;14:337-45.

19. Studýnková JT, Charvát $\mathrm{F}$, Jarošová $\mathrm{K}$, et al. The role of $\mathrm{MRI}$ in the assessment of polymyositis and dermatomyositis. Rheumatology 2007;46:1174-9.

20. Yao L, Gai N. Fat-corrected T2 measurement as a marker of active muscle disease in inflammatory myopathy. Am J Rheumatol 2012;198:W475-81.

21. Degardin A, Morillon D, Lacour A, et al. Morphologic imaging in muscular dystrophies and inflammatory myopathies. Skeletal Radiol 2010;39:1219-27.

22. Hak AE, de Paepe B, de Bleecker JL, et al. Dermatomyositis and polymyositis: new treatment targets on the horizon. Neth $\mathrm{J}$ Med 2011;69:410-21.

23. Selva-O'Callaghan A, Grau JM, Gámez-Cenzano C, et al. Conventional cancer screening versus PET/CT in dermatomyositis/ polymyositis. Am J Med 2010;123:558-62.

24. Morita Y, Kuwagata S, Kato N, et al. ${ }^{18}$ F-FDG PET/CT useful for the early detection of rapidly progressive fatal interstitial lung disease in dermatomyositis. Intern Med 2012;51:1613-18.

25. Jackson RS, Schlarman TC, Hubble WL, et al. Prevalence and patterns of physiologic muscle uptake detected with whole-body ${ }^{18}$ F-FDG PET. J Nucl Med Technol 2006;34:29-33. 